\title{
On the Development of a Rotated-Hybrid HLL/HLLC Approximate Riemann Solver for Relativistic Hydrodynamics
}

\author{
Jamie F. Townsend, ${ }^{1}$ László Könözsy, ${ }^{1 \star}$ and Karl W. Jenkins ${ }^{1}$ \\ ${ }^{1}$ Centre for Computational Engineering Sciences, Cranfield University, Cranfield, MK43 OAL, UK
}

\begin{abstract}
This work presents the development of a rotated-hybrid Riemann solver for solving relativistic hydrodynamics problems with the hybridisation of the HLL and HLLC (or Rusanov and HLLC) approximate Riemann solvers. A standalone application of the HLLC Riemann solver can produce spurious numerical artefacts when it is employed in conjunction with Godunov-type high-order methods in the presence of discontinuities. It has been found that a rotated-hybrid Riemann solver with the proposed HLL/HLLC (Rusanov/HLLC) scheme could overcome the difficulty of the spurious numerical artefacts and presents a robust solution for the Carbuncle problem. The proposed rotated-hybrid Riemann solver provides sufficient numerical dissipation to capture the behaviour of strong shock waves for relativistic hydrodynamics. Therefore, in this work, we focus on two benchmark test cases (odd-even decoupling and double-Mach reflection problems) and investigate two astrophysical phenomena, the relativistic RichtmyerMeshkov instability and the propagation of a relativistic jet. In all presented test cases, the Carbuncle problem is shown to be eliminated by employing the proposed rotated-hybrid Riemann solver. This strategy is problem-independent, straightforward to implement and provides a consistent robust numerical solution when combined with Godunov-type high-order schemes for relativistic hydrodynamics.
\end{abstract}

Key words: hydrodynamics - relativity - shock waves - Riemann solvers - computational methods

\section{INTRODUCTION}

The solution to a local Riemann problem resides at the core of Godunov-type schemes for High-Resolution Shock-Capturing (HRSC) methods. Given two constant initial states between adjacent computational cells, a numerical flux approximation can be obtained via a prescribed Riemann solver. Because the Riemann solver is applied at each cell interface, Godunov-type schemes have a proven track-record of capturing disturbances within the flow field which are treated mathematically as discontinuities. Sudden changes of density, pressure and velocity are thus accommodated in the local flux computations should they exist. This in turn provides excellent shock-capturing qualities when a numerical architecture makes use of this technology. This philosophy has been extensively applied to the field of computational relativistic hydrodynamics (RHD) for special relativity whereby velocities comparable to the speed of light and strong shocks are encountered. High-energy astrophysical phenomena as well as laboratory experiments concerning the quark-gluon plasma (QGP) make extensive use of relativistic hydrodynamics and relativistic magnetohydrodynamics (RMHD) for their physical interpretation.

At present, modern-day finite volume (FV) RHD and RMHD

^ E-mail: laszlo.konozsy@ cranfield.ac.uk (LK) codes can either rely on the exact or approximate solution to the local Riemann problem. The first appearance of an exact Riemann solver for RHD dates back to the analytical solution presented by Martí \& Müller (1994). The solution was then successfully validated using test cases involving strong shocks, ultra-relativistic flows and interacting contact discontinuities, Martí \& Müller (1996). It was then generalised for compatibility to multi-dimensional problems by Pons et al. (2000) such that tangential flow velocities were accommodated. A method to determine the correct wave pattern from the initial conditions before finding the pressure across the contact discontinuity led to the exact Riemann solver of Rezzolla \& Zanotti (2001). The resulting methodology not only proved more efficient but also simpler for numerical implementation.

The Roe Riemann solver, originally developed by Roe (1981), finds the exact solution to the local Riemann problem using a linearised system of equations. This approach was extended to general relativistic flows by Eulderink \& Mellema (1995).

For larger-scale multi-dimensional problems which may also include different multi-physics considerations, approximate Riemann solvers are often preferred. One of the most popular choices of approximate Riemann solver is the HLL solver, originally formulated by Harten et al. (1983) and first applied to relativistic flows by Schneider et al. (1993). Choosing only the smallest and largest wave speeds to determine the bounds of the intermediate state leads 
to a very simple implementation with good performance, Einfeldt (1988). Be that as it may, due to the fact that a contact discontinuity is not resolved, excessive smearing may be present in such cases, Rezzolla \& Zanotti (2013). An even simpler alternative to the HLL Riemann solver is the approximate Riemann solver of Rusanov (1961) that requires only the maximum wave speed in the system. It still, however, possess the disadvantages of the HLL solver due to the inherent larger numerical dissipation. To tackle the issue of excessive smearing found using the HLL approximate Riemann solver, Toro et al. (1994) proposed the HLLC Riemann solver that seeks resolution of the missing contact discontinuity. This proved to be a very powerful strategy and was introduced in the context of RHD by Mignone \& Bodo (2005) and later for RMHD by the same authors in Mignone \& Bodo (2006).

While the HLLC solver provides clear advantages over its predecessor in terms of accuracy due to a more completely resolved wave pattern, issues surround the appearance of the so-called Carbuncle problem under certain flow conditions, see Tsoutsanis (2019). It was reported in Wang et al. (2008) that the relativistic Riemann HLLC solver was in fact "unsuitable" for multi-dimensional problems in RHD due to this numerical artefact. In the realm of classical physics, measures have been taken to improve the robustness of the HLLC Riemann solver using weighted average flux strategies by Kim et al. (2009) and by using selective wave modification by Simon \& Mandal (2019) however the latter requires tuning and may not be applicable as an out-of-the-box solution strategy. The Carbuncle problem resembles itself as a distortion in the flow field when a shock wave is present. It is, in effect, a numerical instability, completely non-physical and detrimental to high-order numerical simulations (see eg. Quirk (1994)). It has been recognised that the appearance of this phenomenon occurs when methods with insufficient numerical dissipation are applied.

As a strategy aimed at keeping the advantages provided by state-of-the-art numerical solutions to the Riemann problem, Nishikawa \& Kitamura (2008) presented the rotated-hybrid Riemann solver concept. This development was based on the rotated Riemann solver approach investigated by Davis (1984); Levy et al. (1993) and Ren (2003) who made use of only a single Riemann solver set to resolve the numerical flux chosen in an adaptively suitable direction. Based on the location of a shock wave or shear layer with respect to a cell boundary, the rotated-hybrid Riemann solver allows for contributions of two base Riemann solvers. By predetermining whether a cell-normal is orthogonal to a shock wave or parallel to a shear layer, a Carbuncle-free base Riemann solver can be applied in that direction. They demonstrated that Carbuncle-free solutions were obtained by this strategy whilst maintaining numerical robustness.

In this report, a direct numerical evaluation of the most popular approximate Riemann solvers is presented. Each approximate Riemann solver is presented in turn followed by a summary of the rotated-hybrid Riemann solver methodology. A rotated-hybrid Riemann solver aimed at circumventing the undesired numerical traits of the relativistic HLLC Riemann solver is then evaluated. Considerations are given to the presence of the Carbuncle problem for flows encountering strong shocks and smooth flow features in which numerical dissipation properties can be directly compared.

\section{GOVERNING EQUATIONS}

The most common description of a relativistic fluid is that of a perfect/ideal fluid. In other words, viscosity and heat conduction are neglected and an ideal equation of state is considered. Throughout this work natural units are used such that the speed of light $c=1$. As is well-known, the equations of motion can be expressed as a system of conservation laws. Their representation within the twodimensional Cartesian coordinate system using the $(3+1)$ "Valencia" formulation yields,

$\frac{\partial}{\partial t} \mathbf{U}+\frac{\partial}{\partial x} \mathbf{F}(\mathbf{U})+\frac{\partial}{\partial y} \mathbf{G}(\mathbf{U})=0$,

where the vector $\mathbf{U}$ is the vector of conserved variables and $\mathbf{F}$ and $\mathbf{G}$ are the flux vectors in the $x$ - and $y$-direction, respectively. The conserved variables are explicitly written as

$\mathbf{U}=\left[\begin{array}{c}D \\ S^{x} \\ S^{y} \\ \tau\end{array}\right]=\left[\begin{array}{c}\rho W \\ \rho h W^{2} u \\ \rho h W^{2} v \\ \rho h W^{2}-p-\rho W\end{array}\right]$,

where $D$ is the rest-mass, $S^{x}$ and $S^{y}$ is the $x$ - and $y$-momentum density, respectively and $\tau$ is the total energy density. These expressions are defined in terms of the primitive variables where $\rho$ is the rest-mass density and $u$ and $v$ denote the fluid velocity in the $x-$ and $y$-direction, respectively. The Lorentz factor, $W$, is expressed as

$W=\frac{1}{\sqrt{1-\left(u^{2}+v^{2}\right)}}$

with the specific enthalpy, $h$, for an ideal gas, given by

$h=1+\epsilon+\frac{p}{\rho}$.

Finally the system is closed with an equation of state of the form $p=p(\rho, \epsilon)$ where $p$ is the pressure and $\epsilon$ is the specific internal energy of the fluid. In the present work, an ideal $\Gamma$-law gas is utilised using a constant adiabatic index, $\Gamma$, which takes the form

$p=\rho \epsilon(\Gamma-1)$.

The primitive variable vector is then defined as

$\mathbf{W}=(\rho, u, v, P)^{T}$.

The corresponding two-dimensional fluxes required to complete the definition of equation (1) are

$\mathbf{F}(\mathbf{U})=\left[\begin{array}{c}D u \\ \rho h W^{2} u^{2}+p \\ \rho h W^{2} u v \\ \rho h W^{2} u^{2}+p-D u\end{array}\right], \quad \mathbf{G}(\mathbf{U})=\left[\begin{array}{c}D v \\ \rho h W^{2} u v \\ \rho h W^{2} v^{2}+p \\ \rho h W^{2} v^{2}+p-D v\end{array}\right]$

The eigenvalues for the system of conservation laws given in the $x$-direction for equation (1) are

$$
\begin{aligned}
\lambda_{0} & =u \\
\lambda_{ \pm} & =\frac{1}{1-v^{2} c_{s}^{2}}\left\{u\left(1-c_{s}^{2}\right)\right. \\
& \left. \pm c_{s} \sqrt{\left(1-v^{2}\right)\left[1-u v-\left(v^{2}-u v\right) c_{s}^{2}\right]}\right\}
\end{aligned}
$$

which determine the wave speeds requisite in the computation of the local time-step and the solution of the local Riemann problem and where $v^{2}=u^{2}+v^{2}$. Through symmetry considerations the eigenvalues in the $y$-direction are easily obtained. The speed of sound, $c_{s}$, is consequently expressed as

$c_{s}^{2}=\frac{\Gamma p}{\rho h}$. 
This completes the description of the necessary governing equations for relativistic hydrodynamics.

\section{NUMERICAL METHODS}

The numerical solution to equation (1) is found by means of a High-Resolution Shock-Capturing (HRSC) Godunov-type method. This is built upon a finite volume (FV) foundation wherein the numerical data is stored at the cell-centre. FV schemes have the advantage of being conservative in nature whilst providing the correct framework for Godunov-type methods that require the solution of the local Riemann problem at each cell boundary. Considering the two-dimensional conservation law in equation (1), a control volume denoted as $\Omega_{i, j}=\left[x_{i-\frac{1}{2}}, x_{i+\frac{1}{2}}\right] \times\left[y_{j-\frac{1}{2}}, y_{j+\frac{1}{2}}\right]$ is defined to be integrated over. This operation then yields

$$
\frac{d \mathbf{u}_{i, j}}{d t}=-\frac{\mathbf{f}_{i+\frac{1}{2}, j}-\mathbf{f}_{i-\frac{1}{2}, j}}{\Delta x}-\frac{\mathbf{g}_{i, j+\frac{1}{2}}-\mathbf{g}_{i, j-\frac{1}{2}}}{\Delta y}
$$

where $\mathbf{u}_{i, j}$ is the spatial average in the cell $\Omega_{i, j}$ at a time $t$ and the fluxes $\mathbf{f}_{i \pm \frac{1}{2}, j}$ and $\mathbf{g}_{i, j \pm \frac{1}{2}}$ correspond to the average spatial fluxes over the cell boundaries $x_{i \pm \frac{1}{2}, j}$ and $y_{i, j \pm \frac{1}{2}}$, respectively. The fluxes are then computed by an appropriate Riemann solver, discussed in section 4 , using values that arise from a spatial reconstruction scheme.

To obtain the initial conditions required for the numerical solution of the Riemann problem a spatial reconstruction strategy is required. In the present research, the WENO (Weighted Essentially Non-Oscillatory) concept is applied to the primitive variables, see Shu (2009). WENO schemes are well-suited for problems containing discontinuities and complex smooth flow features. WENO methods use a combination of adaptive stencils, each assigned a specific non-linear weight depending on the local smoothness surrounding each stencil. When a stencil contains non-smooth data, i.e. a discontinuity, its weight are set to, essentially, zero. On the other hand, when a solution contains smooth data, optimal weights are prescribed. The order of accuracy for WENO schemes is determined by the number of cells in each sub-stencil, $r$, such that the entire reconstruction polynomial is of the order $(2 r-1)$. For more information regarding WENO reconstruction the reader is referred to Titarev \& Toro (2004); Shu (2009); Balsara (2017).

A common numerical practice when high-order WENO schemes are used is to reduce the order of accuracy and thus the stencil size in regions surrounding shocks. This aids in alleviating the so-called Gibbs phenomenon which gives rises to oscillations in the solution, see Nunez-de la Rosa \& Munz (2016). The shock detection itself can be achieved using the shock indicator of Jameson et al. (1981),

$\eta_{i}=\frac{\left|p_{i+1}-2 p_{i}+p_{i-1}\right|}{\left|p_{i+1}\right|+2\left|p_{i}\right|+\left|p_{i-1}\right|}$

where $p$ is the fluid pressure. Should the value of $\eta$ exceed $\eta=$ $5 \times 10^{-3}$ a shock is said to be detected in the neighboring region and a lower order scheme can be temporarily deployed; this is found to improve the numerical stability of the solution.

An explicit third-order TVD (Total Variation Diminishing) Runge-Kutta (RK3) method integrates the numerical solution in time. This can be summarised through the three stages:

$\mathbf{U}^{(1)}=\mathbf{U}^{n}+\Delta t Q\left(\mathbf{U}^{n}\right)$,

$\mathbf{U}^{(2)}=\frac{1}{4}\left[3 \mathbf{U}^{n}+\mathbf{U}^{(1)}+\Delta t Q\left(\mathbf{U}^{(1)}\right)\right]$,

$\mathbf{U}^{(3)}=\frac{1}{3} \mathbf{U}^{n}+\frac{2}{3} \mathbf{U}^{(2)}+\frac{2}{3} \Delta t Q\left(\mathbf{U}^{(2)}\right)$

where $Q$ denotes the numerical flux to be determined from the Riemann solver stage. For the RK3 method to remain TVD an appropriate time-step $\Delta t$ must be chosen, Gottlieb \& Shu (1998). For a two-dimensional system $(\operatorname{Dim}=2)$ this can be computed from

$\Delta t=\frac{\mathrm{CFL}}{\operatorname{Dim}} \min \left(\frac{\Delta x}{\left|\lambda_{x}\right|}, \frac{\Delta y}{\left|\lambda_{y}\right|}\right)$

where $\left|\lambda_{x, y}\right|$ represents the maximum signal speed in either the $x-$ or $y$-direction provided from equation (8) and CFL denotes the stability criterion which follows the constraint $\mathrm{CFL} \leq 1$.

The conserved variables are the variables evolved in time by equation (12) and therefore a procedure is required to obtain the primitive variables that are of interest. Unlike in classical hydrodynamics, a closed-form mapping between the conserved and primitive variables does not exist and therefore an additional amount of numerical effort is required. A root-finding algorithm must be used in the recovery procedure and the reader is referred to Appendix D of Rezzolla \& Zanotti (2013) for details of the scheme used in the present research. A convergence criterion is established using the $L_{2}$ error norm for the pressure term with a tolerance of $10^{-8}$; this typically convergences within 11 iterations or fewer.

The methods described in this section have been show to provide accurate and robust solutions for the RHD equations and comprise the methods used in this research. For more details about their use and other methods for the numerical solution of the RHD equations within the HRSC framework the reader is referred to Martí \& Müller (2015) for an up-to-date review.

\section{RIEMANN SOLVERS}

The numerical fluxes needed to be found in equation (10) are obtained via the solution of a local Riemann problem at a cell boundary. The flux $\mathbf{f}_{i-\frac{1}{2}}$ for example is found from initial data

$\mathbf{U}(x, 0)= \begin{cases}\mathbf{U}_{L, i-\frac{1}{2}} & \text { if } x<x_{i-\frac{1}{2}} \\ \mathbf{U}_{R, i-\frac{1}{2}} & \text { if } x>x_{i-\frac{1}{2}}\end{cases}$

where $\mathbf{U}_{L, i-\frac{1}{2}}$ and $\mathbf{U}_{R, i-\frac{1}{2}}$ are the values to the left and to the right of the cell boundary, respectively. The numerical flux across that boundary is computed by means of an exact or approximate Riemann solver. In the following sub-sections, the most popular approximate Riemann solvers for RHD: the Rusanov, HLL and HLLC Riemann solvers are introduced followed by the implementation strategy for the rotated-hybrid Riemann solver.

\subsection{The HLL Riemann Solver}

A popular choice for the solution of the local Riemann problem is the HLL Riemann solver of Harten et al. (1983), later modified by Einfeldt (1988) to yield the HLLE approximate Riemann solver. Its simple implementation and proven track-record of reliable flux computations have earnt it the position of the standard workhorse within the majority of RHD research codes. 
The HLLE Riemann solver, hereafter HLL, assumes that after the initial discontinuity decay, only two waves emanate that propagate in opposite directions with velocities $S_{L}$ and $S_{R}$ leaving a constant single-state in-between. With knowledge of the smallest and largest characteristic wave speeds, i.e. $S_{L} \leq 0$ and $S_{R} \geq 0$, the numerical flux at the cell boundary can be computed via

$\mathbf{F}_{i-\frac{1}{2}}= \begin{cases}\mathbf{F}_{L} & \text { if } 0 \leq S_{L}, \\ \mathbf{F}^{\mathrm{HLL}} & \text { if } S_{L} \leq \leq S_{R}, \\ \mathbf{F}_{R} & \text { if } 0 \geq S_{R},\end{cases}$

where $\mathbf{F}_{L}=\mathbf{F}\left(\mathbf{U}_{L}\right), \mathbf{F}_{R}=\mathbf{F}\left(\mathbf{U}_{R}\right)$ and $\mathbf{F}^{\mathrm{HLL}}$ is the numerical HLL flux in the constant intermediate state given by

$\mathbf{F}^{\mathrm{HLL}}=\frac{S_{R} \mathbf{F}_{L}-S_{L} \mathbf{F}_{R}+S_{L} S_{R}\left(\mathbf{U}_{R}-\mathbf{U}_{L}\right)}{S_{R}-S_{L}}$.

A further comment should be made about the choice of wave speeds. $S_{L}$ and $S_{R}$ should be chosen accordingly via

$S_{L}:=\min \left(0, S_{-}\left(\mathbf{U}_{L}\right), S_{-}\left(\mathbf{U}_{R}\right)\right)$,

$S_{R}:=\max \left(0, S_{+}\left(\mathbf{U}_{L}\right), S_{+}\left(\mathbf{U}_{R}\right)\right)$,

where $S_{-}$and $S_{+}$are provided by the expressions in equation (8).

The HLL approximate Riemann solver is a robust strategy for numerical flux computations, is exact for single shocks and demonstrates good performance at critical sonic rarefactions, Rezzolla \& Zanotti (2013); Martí \& Müller (2015). On the other hand, in areas surrounding contact discontinuities, it is very dissipative. This is because the middle wave in the Riemann fan is ignored which subsequently prohibits the correct wave pattern when a contact discontinuity is present in the solution. The HLLC approximate Riemann solver presented in 4.3 offers a remedy to this issue.

\subsection{The Rusanov Riemann Solver}

Another simple numerical flux implementation is the Riemann solver of Rusanov (1961). Unlike the HLL Riemann solver only one wave speed is required for the numerical flux computations, this is found from, $S=\max \left(\left|S_{L}\right|,\left|S_{R}\right|\right)$, where $S_{L}$ and $S_{R}$ and given by (17). The numerical flux is then computed via

$\mathbf{F}^{\text {Rusanov }}=\frac{1}{2}\left(\mathbf{F}_{L}+\mathbf{F}_{R}\right)-\frac{1}{2} S\left(\mathbf{U}_{R}-\mathbf{U}_{R}\right)$.

Similarly to the HLL solver, the Rusanov solver also has the property of high numerical dissipation. That being said, when the HLL and Rusanov solvers are used in conjunction with high-order spatial reconstruction, troublesome numerical artefacts such as the Carbuncle phenomenon can be avoided, see e.g. Quirk (1994); Maccormack (2011).

\subsection{The HLLC Riemann Solver}

The HLLC approximate Riemann solver, where 'C' stands for contact, was introduced by Toro et al. (1994) to resolve the missing intermediate contact discontinuity absent in the HLL construction. Within the relativistic framework the HLLC solver was reformulated firstly for RHD by Mignone \& Bodo (2005) and later for RMHD by the same authors, Mignone \& Bodo (2006). The implementation strategy adopted closely follows Mignone \& Bodo (2005) and is summarised here for the $x$-direction.
Firstly the HLL state and flux vectors must be computed,

$\mathbf{U}_{\mathrm{HLL}}=\left[\begin{array}{c}D_{\mathrm{HLL}} \\ S_{\mathrm{HLL}}^{x} \\ S_{\mathrm{HLL}}^{y} \\ \tau_{\mathrm{HLL}}\end{array}\right]=\frac{S_{R} \mathbf{U}_{R}-S_{L} \mathbf{U}_{L}+\mathbf{F}_{L}-\mathbf{F}_{R}}{S_{R}-S_{L}}$,

along with the corresponding HLL fluxes,

$\mathbf{F}_{\mathrm{HLL}}=\left[\begin{array}{c}F_{\mathrm{HLL}}^{D} \\ F_{\mathrm{HL}}^{S^{x}} \\ F_{\mathrm{HLL}}^{S^{y}} \\ F_{\mathrm{HLL}}^{\tau}\end{array}\right]=\frac{S_{R} \mathbf{F}_{L}-S_{L} \mathbf{F}_{R}+S_{L} S_{R}\left(\mathbf{U}_{R}-\mathbf{U}_{L}\right)}{S_{R}-S_{L}}$.

The intermediate wave speed in the $x$-direction is found via the quadratic equation,

$\left(F_{\mathrm{HLL}}^{\tau}+F_{\mathrm{HLL}}^{D}\right)\left(S^{*}\right)^{2}-\left[(\tau+D)+F_{\mathrm{HLL}}^{S^{x}}\right] S^{*}+S^{x}=0$.

The positive root of the quadratic formula then provides a suitable intermediate wave speed, $S^{*}$. By consideration of the relativistic Rankine-Hugonoit conditions the remainder of the conserved quantities within the intermediate star region can be obtained in each spatial direction,

$\mathbf{F}_{L_{*}}=\mathbf{F}_{L}+S_{L}\left(\mathbf{U}_{L_{*}}-\mathbf{U}_{L}\right)$,

$\mathbf{F}_{R_{*}}=\mathbf{F}_{R}+S_{R}\left(\mathbf{U}_{R_{*}}-\mathbf{U}_{R}\right)$.

The corresponding HLLC numerical fluxes are then given by,

$\mathbf{F}_{i-\frac{1}{2}}= \begin{cases}\mathbf{F}_{L} & \text { if } S_{L} \geq 0, \\ \mathbf{F}_{L}^{*} & \text { if } S_{L} \leq 0 \leq S^{*}, \\ \mathbf{F}_{R}^{*} & \text { if } S^{*} \leq 0 \leq S_{R}, \\ \mathbf{F}_{R} & \text { if } S_{R} \leq 0,\end{cases}$

The HLLC Riemann solver is capable of providing accurate resolution of shocks in one-dimensional problems. In general, it produces a more accurate restoration of the contact and tangential discontinuities in comparison with the HLL Riemann solver. However, as discussed by Wang et al. (2008), numerical pathologies in higherdimensions may emerge when shocks are present in the solution.

\section{THE ROTATED-HYBRID RIEMANN SOLVER}

The solution methodology for the rotated-hybrid Riemann solver closely follows that of Nishikawa \& Kitamura (2008). The motivation for including the rotated-hybrid Riemann solver philosophy is also identical, that is, to increase robustness of the numerical solution of the RHD equations given by equation (1). For completeness their strategy is summarised which can be easily implemented using the aforementioned base Riemann solvers for RHD presented in section 4 . The rotated-hybrid Riemann solver requires a definition of a normal vector $\mathbf{n}$ with respect to a given cell-face. This vector is built such that it can be decomposed into two orthogonal directions, $\mathbf{n}_{1}$ and $\mathbf{n}_{2}$. By definition $\mathbf{n}_{1} \cdot \mathbf{n}_{2}=0$ for any choice of $\mathbf{n}$. In addition, $\left|\mathbf{n}_{1}\right|=\left|\mathbf{n}_{2}\right|=1$ is imposed, see figure 1 . The projection of the cell-face normal onto the two orthogonal vectors provides,

$\mathbf{n}=\alpha_{1} \mathbf{n}_{1}+\alpha_{2} \mathbf{n}_{2}$,

where the scalar values $\alpha_{1}$ and $\alpha_{2}$ are computed from

$\alpha_{1}=\mathbf{n} \cdot \mathbf{n}_{1}$,

$\alpha_{2}=\mathbf{n} \cdot \mathbf{n}_{2}$. 


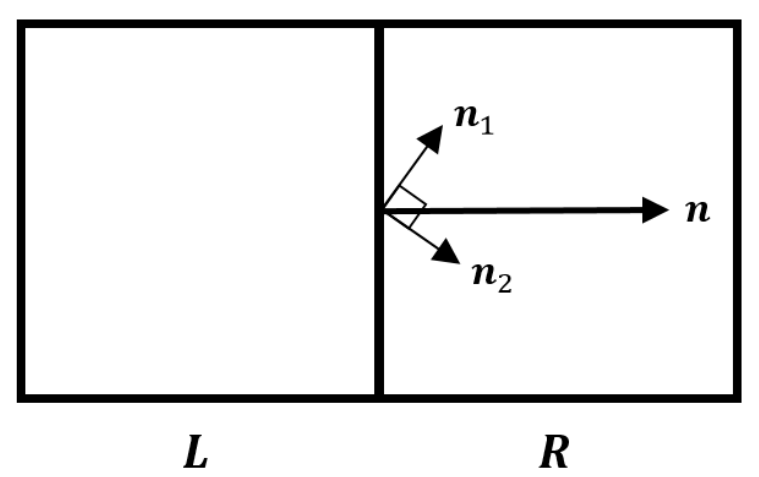

Figure 1. Definition of vector components for the rotated-hybrid Riemann solver.

The numerical flux for the rotated-hybrid concept is then formulated based on the decomposition given by,

$\mathbf{F}(\mathbf{n})=\alpha_{1} \mathbf{F}\left(\mathbf{n}_{1}\right)+\alpha_{2} \mathbf{F}\left(\mathbf{n}_{2}\right)$.

Two independent Riemann solvers can therefore be utilised for the flux computation in directions $\mathbf{n}_{1}$ and $\mathbf{n}_{2}$. The amount of weight placed on each flux contribution is specified by the coefficients $\alpha_{1}$ and $\alpha_{2}$ which is determined by the direction of vector $\mathbf{n}_{1}$ at the cell boundary. This concept allows a Riemann solver to be applied in a direction not restricted by the grid geometry, contrary to standard Riemann solver implementations on a structured Cartesian grid. Following Levy et al. (1993), the vector $\mathbf{n}_{1}$ is computed using velocity differencing such that it directs either in the direction normal to a shock or parallel to a shear layer,

$\mathbf{n}_{1}=\left\{\begin{array}{cl}\frac{\Delta \vec{q}}{\|\Delta \vec{q}\|}, & \text { if }\|\Delta \vec{q}\| \geq \epsilon, \\ \mathbf{n}, & \text { otherwise. }\end{array}\right.$

The velocity difference vector $\Delta \vec{q}$ and its modulus are defined by

$$
\begin{aligned}
\Delta \vec{q} & =(\Delta u, \Delta v)=\left(u_{R}-u_{L}, v_{R}-v_{L}\right), \\
\|\Delta \vec{q}\| & =\sqrt{(\Delta u)^{2}+(\Delta v)^{2}}
\end{aligned}
$$

and $\epsilon$ is a small number set to $\epsilon=10^{-12}\left(1+U_{\text {ref }}\right)$ in the present work, where $U_{\text {ref }}$ is a reference velocity magnitude. Should a shock and a shear layer be present at the cell-interface then the shock will propagate in the direction of $\mathbf{n}_{1}$ whilst the shear layer will travel in the direction of $\mathbf{n}_{2}$, Ren (2003). Furthermore, if a strong rarefaction wave is to exist then this would also invoke the application of the dissipative Riemann solver. This choice of $\mathbf{n}_{1}$ is designed to ensure that sufficient numerical dissipation is applied in the vicinity of shocks to avoid the Carbuncle phenomenon and ensure that excessive numerical dissipation is not present in shear layers to ensure important flow features are not artificially smoothed out. The vector $\mathbf{n}_{2}$ is then consequently specified to be perpendicular to $\mathbf{n}_{1}$ and the scalar values $\alpha_{1}$ and $\alpha_{2}$ are then found from the equations in (25) whereby $\alpha_{1} \geq 0$ and $\alpha_{2} \geq 0$.

An alternative choice of $\mathbf{n}_{1}$ consists of defining the flux in the tangential direction to a shear layer such that the HLLC Riemann solver is also deployed if the streamwise velocity is smooth. In other words, the dissipative Riemann solver is turned off in regions surrounding shear layers to recover only the numerical flux arising from the HLLC Riemann solver. This modification of $\mathbf{n}_{1}$ is made such that

$\mathbf{n}_{1}=\left\{\begin{array}{cl}\frac{\Delta \vec{q}}{\|\Delta \vec{q}\|}, & \text { if }\|\Delta \vec{q}\| \geq \epsilon, \\ \mathbf{n}_{\perp}, & \text { otherwise }\end{array}\right.$ where $\mathbf{n}_{\perp}$ is the tangential direction to the geometric face normal. It is found that, in general, this alternative formulation has only a small influence on the solution for the test cases considered. This is to be somewhat expected as it is the cross-diffusion rather than the streamwise diffusion that is known to have a greater impact on the numerical solution at shear layers, Nishikawa \& Kitamura (2008). Unless otherwise state, the definition of $\mathbf{n}_{1}$ will always be chosen as that in equation (27).

With the rotated-hybrid Riemann solver concept described it is now possible to amalgamate a choice of base Riemann solvers to complete the numerical implementation. In this work, the HLLC is chosen as primary Riemann solver and the Rusanov or HLL approximate Riemann solvers are adopted in the troublesome direction perpendicular to a shock. In general, the rotated-hybrid Riemann solver can be constructed via,

$\mathbf{F}=\alpha_{1} \mathbf{F}_{\mathrm{HLL}}\left(\mathbf{n}_{1}\right)+\alpha_{2} \mathbf{F}_{\mathrm{HLLC}}\left(\mathbf{n}_{2}\right)$,

$\mathbf{F}=\alpha_{1} \mathbf{F}_{\text {Rusanov }}\left(\mathbf{n}_{1}\right)+\alpha_{2} \mathbf{F}_{\text {HLLC }}\left(\mathbf{n}_{2}\right)$,

for the rotated-hybrid HLL/HLLC and Rusanov/HLLC formulations, respectively. The dissipative (HLL or Rusanov) Riemann solver acts predominantly in the direction normal to a shock or parallel to shear layers, should they exist, based on the definition of $\mathbf{n}_{1}$ from (27) whilst the HLLC Riemann solver is used in all other regions of the flow. If the direction of $\mathbf{n}_{1}$ is chosen from equation (29) then the HLLC Riemann solver will be utilised parallel to a shear layer if the streamwise velocity is smooth.

This implementation is simple, straightforward to implement and is independent of problem-specific parameters meaning that no $a d$-hoc configuration is required for different relativistic problems.

\section{RESULTS}

To assess the performance of the rotated-hybrid Riemann solver methodology within the context of relativistic hydrodynamics, four test cases known to produce numerical pathologies are presented. The first two are purely numerical cases, the shock-instability problem in section 6.1 and the relativistic double-Mach reflection in section 6.2. These cases are widely known to be negatively affected by full-wave and exact Riemann solvers resulting in the appearance of the Carbuncle phenomenon. The second two cases are applicable to those concerned with the study of high-energy astrophysical problems. The relativistic Richtmyer-Meshkov instability in section 6.3 and a relativistic jet in section 6.4 are considered and shown that their numerical simulation can also be hindered by Carbuncle artefacts.

\subsection{The Shock-Instability (Odd-Even Decoupling) Problem}

Insight into whether or not a Riemann solver is susceptible to the Carbuncle instability is provided by Liou (2000) who investigated the numerical diffusivity of various numerical flux schemes. Liou states that the mass flux can be written in the general form

$\dot{\mathbf{m}}=\langle\dot{\mathbf{m}}\rangle-\frac{1}{2} \mathcal{D}\left(\mathbf{U}_{L}, \mathbf{U}_{R}\right)$

in which $\langle\dot{\mathbf{m}}\rangle$ is a centrally weighted average that can be simply expressed by

$\langle\dot{\mathbf{m}}\rangle=\frac{1}{2}\left[(\rho u W)_{L}+(\rho u W)_{R}\right]$ 
in the context of the ideal RHD equations. $\mathcal{D}$ is defined as the numerical dissipation term which can be expressed as differences of primitive variables. Considering only $\rho, u$ and $p$ one would find,

$\mathcal{D}=\mathcal{D}^{(\rho)}(\overline{\mathbf{U}}) \Delta \rho+\sum_{h} \mathcal{D}^{\left(u_{h}\right)}(\overline{\mathbf{U}}) \Delta u_{h}+\mathcal{D}^{(p)}(\overline{\mathbf{U}}) \Delta p$

which enables the extraction of dissipative contributions from those primitive variables. Liou determined that a pressure contribution in the dissipative part of the mass flux (continuity) equation relating to the numerical scheme is the culprit for this numerical instability. In other words, schemes that have the property $\mathcal{D}^{(p)}=0$ in the mass flux are shock-stable and are not prone to this numerical instability. To illustrate ideas, the dissipative pressure term $\mathcal{D}^{(p)}$ is analysed for the relativistic HLL and HLLC Riemann solvers in turn. The HLL flux can be written as a single formula as

$$
\mathbf{F}^{\mathrm{HLL}}=\frac{1}{2}\left[\mathbf{F}_{L}+\mathbf{F}_{R}\right]+\underbrace{\frac{S_{R} S_{L}}{S_{R}-S_{L}} \Delta \mathbf{U}-\frac{1}{2} \frac{S_{R}+S_{L}}{S_{R}-S_{L}} \Delta \mathbf{F}}_{\text {Dissipation Terms }},
$$

where the structure of the flux contains a contribution from the mass flux and dissipative terms. Using the expressions in (2) and (7) for the RHD governing equations in the $x$-direction the above formula becomes,

$$
\begin{aligned}
\mathbf{F}_{\text {cont. }}^{\mathrm{HLL}}=\frac{1}{2}\left[(\rho u W)_{L}+\right. & \left.(\rho u W)_{R}\right]+\frac{S_{R} S_{L}}{S_{R}-S_{L}}\left[(\rho W)_{R}-(\rho W)_{L}\right] \\
& -\frac{1}{2} \frac{S_{R}+S_{L}}{S_{R}-S_{L}}\left[(\rho u W)_{R}-(\rho u W)_{L}\right] .
\end{aligned}
$$

By inspection it is possible to see from (35) that there are no pressure difference terms in the continuity equation for the HLL scheme and therefore $\mathcal{D}^{(p)}=0$ and the scheme is shock-stable. The HLLC Riemann solver can be re-expressed as a single formula, see Kim et al. (2010),

$\mathbf{F}^{\mathrm{HLLC}}=C_{1} \mathbf{F}_{L}+C_{2} \mathbf{F}_{R}+A\left(\mathbf{U}_{L}^{*}-\mathbf{U}_{L}\right)+B\left(\mathbf{U}_{R}^{*}-\mathbf{U}_{R}\right)$

where $C_{1}, C_{2}, A$ and $B$ are given in Kim et al. (2010) as,

$$
\begin{aligned}
C_{1} & =\frac{\max \left(0, S^{*}\right)}{S^{*}} ; \quad C_{2}=\frac{\min \left(0, S^{*}\right)}{S^{*}}, \\
A & =S_{L}\left(C_{1}-\frac{\max \left(0, S_{L}\right)}{S_{L}}\right) ; \quad B=S_{R}\left(C_{2}-\frac{\min \left(0, S_{R}\right)}{S_{R}}\right) .
\end{aligned}
$$

The mass flux for the ideal RHD equations can then be analysed through use of (36),

$$
\begin{aligned}
\mathbf{F}_{\text {cont. }}^{\mathrm{HLLC}}=C_{1}(\rho u W)_{L}+C_{2}(\rho u W)_{R} \\
+\underbrace{A(\rho u W)_{L} \frac{S_{*}-u_{L}}{S_{L}-S_{*}}+B(\rho u W)_{R} \frac{S_{*}-u_{R}}{S_{R}-S_{*}}}_{\text {Dissipation Term }},
\end{aligned}
$$

which allows for the numerical dissipation terms to be isolated,

$\mathcal{D}=A(\rho u W)_{L} \frac{S_{*}-u_{L}}{S_{L}-S_{*}}+B(\rho u W)_{R} \frac{S_{*}-u_{R}}{S_{R}-S_{*}}$.

Considering only the contributions of pressure in (39) it can be seen that differencing in $p$ is not explicitly present. In the case of classical hydrodynamics, the intermediate wave speed $S_{*}$ is found from an expression that contains differencing in $p$. However, when $S_{*}$ is computed in the RHD case it is found from the solution of a quadratic equation (21). Within equation (21) are the HLL fluxes found from equation (20) which include differences in left and right conserved variables, which means pressure differencing is manifest in the calculation of the intermediate wave speed and therefore $\mathcal{D}^{(p)} \neq 0$. This then implies by the logic presented by Liou (2000) that the relativistic HLLC Riemann solver of Mignone \& Bodo (2005) is shock unstable.

To evaluate the shock instability resulting from the relativistic HLLC Riemann solver the odd-even decoupling problem is considered. First introduced by Quirk (1994), the odd-even decoupling problem consists of perturbing an otherwise uniform grid such that a disjointed cross-section coincides with a moving shock wave. Instead of a clean shock profile, oscillations surrounding the shock interface emerge in the density field, which eventually deteriorate the solution. This well-known shock instability has been reported within the classical literature for the Roe and HLLC Riemann solvers, see Quirk (1994); Ren (2003); Kim et al. (2009); Simon \& Mandal (2019).

Since the data structure of the presented solver does not permit grid perturbations an alternative demonstration is presented to evaluate the relativistic Riemann solvers. Given that the numerical dissipation term $\mathcal{D}^{(p)}$ is used to explain the appearance of the Carbuncle phenomenon, an analogue of the original odd-even decoupling problem is presented. Quirk (1994) showed theoretically how perturbations in the pressure field, however small, can grow linearly to influence the density and velocities fields to corrupt the solution. Hence, a test case consisting of a pressure field perturbation that disturbs the moving shock is presented. A two-dimensional channel of size $[0,16] \times[0,0.5]$ contains a rightward propagating shock initially positioned at $x=0.1$. The left boundary condition is designated as an inflow with Dirichlet conditions prescribed as those behind the shock. The right boundary is prescribed as a Neumann-type outflow whilst the top and bottom boundaries are periodic. The initial primitive variable conditions (see equation (6)) ahead and behind the shock are denoted with subscript $a$ and $b$, respectively, and are given by:

$\mathbf{W}_{a}=(1.0,0.0,0.0,0.1)^{T}$,

$\mathbf{W}_{b}=(6.59,0.748,0.0,2.4)^{T}$.

The initial conditions of the shock are such that the shock propagates with a velocity $v_{s}=0.831$ with corresponding Lorentz factor $W_{s}=$ 1.8 and relativistic Mach number $M_{S}=4.61$. A small perturbation is applied to the pressure field $p_{i, j}$ at a position ahead of the initial shock at $i=100$ as follows

$p_{100, j}= \begin{cases}p_{j}=0.1+\hat{p}, & \text { if } j=\text { even }, \\ p_{j}=0.1, & \text { otherwise, }\end{cases}$

where $\hat{p}$ is chosen to be 0.01 and a grid resolution of $800 \times 40$ computational cells is used. Simulations are then performed until time $t=18$ for the HLL, HLLC, rotated-hybrid HLL/HLLC and rotated-hybrid Rusanov/HLLC Riemann solvers. Figure 2 reports the condition of the incident shock as it propagates through the domain at different times with the different single and rotated-hybrid Riemann solver configurations. Only for these test cases, a firstorder Runge-Kutta method (also known as the Euler method) is used for time integration and a first-order spatial reconstruction scheme is used - this ensures that any numerical dissipation arises predominantly from the chosen Riemann solver and not the spatial reconstruction or time integration method. The HLL Riemann solver in figures $2 \mathrm{a}-2 \mathrm{a}$ yields a clean and consistent shock profile at all times in the simulation. The HLLC Riemann solver on the other hand in figures $2 \mathrm{a}-2 \mathrm{a}$ shows a disturbed solution. The incident shock experiences fluctuations in the rest-mass density field behind the shock caused by the pressure perturbation which worsens over time. When, on the other hand, the rotated-hybrid method is used, 
it can be seen in figures $2 \mathrm{~g}-2 \mathrm{l}$ that this numerical instability is avoided. Because the HLL or Rusanov Riemann solver provides the dominant flux contribution in the vicinity of the shock the inherent numerical dissipation is enough to overcome the growth of the pressure perturbations in a consistent manner.

\subsection{The Relativistic Double-Mach Reflection Problem}

To evaluate the credibility of a numerical solver designed to accurately capture shock waves, the double-Mach reflection test case must be a component in the numerical test bench. Originally devised by Woodward \& Colella (1984) and later applied to RHD by Zhang \& MacFadyen (2006), the test sees an incident shock wave with a $60^{\circ}$ angle to the $x$-axis propagate rightward through the domain. The shock then interacts with a reflective boundary condition imposed along the lower $x$-axis at $x \geq 1 / 6$. At the left boundary, an inflow boundary condition is implemented that mimics the conditions behind the shock and an outflow boundary condition is prescribed at the right domain boundary. The top boundary condition is set to either a pre- or post-shock value depending on the shock position. The computational domain is of size $[0,4] \times[0,1]$ and is discretised by $1600 \times 400$ computational cells; for all test cases the WENO5 scheme is employed. All simulations are run for a total time of $t=4$ and the initial conditions are specified in terms of the primitive variables defined in equation (6). Following Nunez-de la Rosa \& Munz (2016), the initial conditions are given by

$\mathbf{W}_{L}=\left(8.564,0.4247 \sin 60^{\circ},-0.4247 \cos 60^{\circ}, 0.3808\right)^{T}$,

$\mathbf{W}_{R}=(1.4,0.0,0.0,0.0025)^{T}$

with an adiabatic index of $\Gamma=7 / 5$. The beginning of the reflecting wall, denoted $x_{0}$, and the exact solution of the shock wave position, $h(x, t)$ travelling with velocity $v_{s}$ is given by

$h(x, t)=\sqrt{3}\left(x-x_{0}\right)-2 v_{s} t, \quad x_{0}=\frac{1}{6}, \quad v_{s}=0.4984$

which must be considered for the correct implementation of the top boundary condition.

The results presented within figure 3 showcase the rest-mass density contours for the relativistic double-Mach reflection with the standard base Riemann solvers and their rotated-hybrid modifications. Figure 3a provides an overview of the complete problem whereas figures $3 b-3 d$ show only the portion of the domain in the interaction zone. The appearance of a kinked Mach stem, seen in figure $3 \mathrm{~b}$ when the HLLC Riemann is employed, is an indication of the numerical scheme's sensitivity to the Carbuncle instability. It has been reported in the classical literature that use of the Roe and HLLC Riemann solvers can result in the appearance of a kinked Mach stem (see e.g. Quirk (1994); Tsoutsanis (2019)) and the same finding can now be seen when the relativistic HLLC Riemann solver of Mignone \& Bodo (2005) is in use. Note that when the relativistic HLL Riemann solver is used then a kinked Mach stem is not present. This extends to the rotated-hybrid Riemann solvers seen in figures $3 \mathrm{c}$ and $3 \mathrm{~d}$ that do not display a kinked Mach stem due to the HLL or Rusanov Riemann solver providing the dominant flux contribution in the direction normal to the shock and adequate numerical dissipation in the region of the angled shock.

\subsection{Relativistic Richtmyer-Meshkov Instability}

The Richtmyer-Meshkov instability (RMI) occurs when an impulsive acceleration, such as a shock wave, is applied across a perturbed interface with contrasting density. This instability was theoretically predicted by Richtmyer (1960) and later experimentally confirmed by Meshkov (1972). Unlike the Rayleigh-Taylor instability (RTI) which develops under the condition of constant acceleration directed towards the lighter fluid, the RMI can develop when such an impulsive acceleration is directed towards either the heavy or lighter fluid. As the incident shock wave initially coincides with the interface the perturbation is compressed. This is then followed by a period of linear growth as the shock wave drags the interface rightward leaving a trailing spike. A velocity shear is generated along the spike and eventually a transition to turbulence and subsequent mixing occur. While the vast majority of studies concerning the RMI are centred around its application to inertial confinement fusion (ICF), applications within high-energy astrophysical environments are also of important scientific interest. Inoue (2012) has considered implications of the RMI in the context of RMHD for pulsar wind nebulae, gamma-ray bursts and active galactic nuclei, Matsumoto \& Masada (2013) studied the role of the RTI and RMI on the structure of relativistic jets and Kane et al. (1999) evaluates what role the RMI plays in supernova remnant formation.

To date, only a handful of studies have been conducted that seek understanding of the RMI in conjunction with relativistic effects. Initially Mohseni et al. (2014) studied the relativistic RMI via a particle-based solver. Most notably however was the extension of the previous work and subsequent confirmation of RMI suppression due to Lorentz contraction as the Lorentz factor of the incident shock exceeded a critical value by Zanotti \& Dumbser (2015). Following Zanotti \& Dumbser (2015), simulations of the relativistic RMI are performed and the prescribed Riemann solver is varied between the HLL, HLLC and the rotated-hybrid HLL/HLLC Riemann solvers.

Figure 4 shows a schematic diagram representing the initial conditions of the relativistic Richtmyer-Meshkov instability. The total size of the domain is $[0,15] \times[0,2.5]$ where a rightward propagating shock is placed initially at $x=1.0$. Ahead of the shock a perturbed interface separates a discontinuous change in rest-mass density given by

$x=x_{0}+a \sin \left(\frac{\pi}{2}+\frac{2 \pi y}{\lambda}\right)$

where $a=0.1$ is the perturbation amplitude, $x_{0}=3$ and $\lambda=2.5$ corresponds to the perturbation wavelength. Initial conditions left of the interface are denoted with the subscript $L$ whereas conditions to the right are denoted with the subscript $R$. Similarly, initial conditions behind the shock are denoted with subscript $b$. The initial conditions are specified in terms of the primitive variables defined in equation (6) and are given by

$\mathbf{W}_{b}=(5.13,0.633,0.0,1.35)^{T}$,

$\mathbf{W}_{L}=(1.0,0.0,0.0,0.1)^{T}$,

$\mathbf{W}_{R}=(35.0,0.0,0.0,0.1)^{T}$.

This problem setup leads to a positive Atwood number configuration of $A=0.98$ in which the shock propagates rightward with relativistic Mach number 3.44 and incident Lorentz factor 1.5. Periodic boundary conditions are used for the top and bottom boundaries, the left boundary is prescribed as an inflow, set to the conditions behind the shock, whilst the right boundary is prescribed as an outflow. The computational domain is discretised with $1500 \times 400$ cells unless stated otherwise and an adiabatic index of $\Gamma=4 / 3$ is used throughout.

Figure 5 provides a snapshot of the growing RMI at $t \approx 30$, highlighting the difference between the Rusanov, HLL, HLLC and 


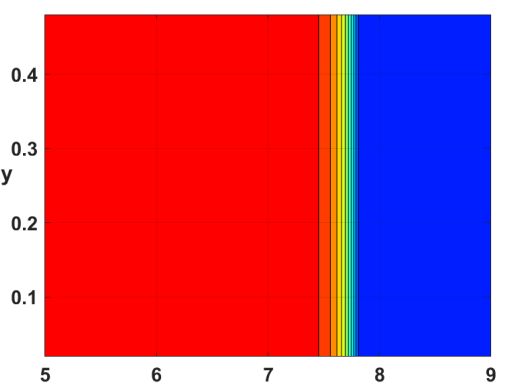

(a) Standard HLL: $t \approx 9.0$

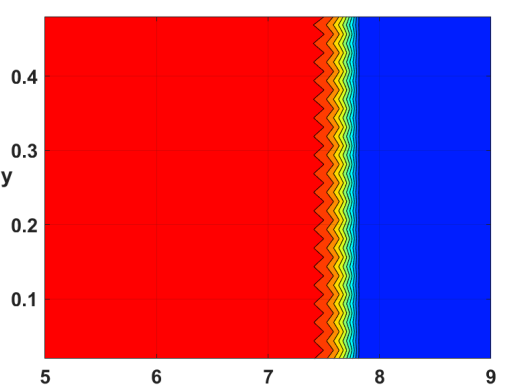

(d) Standard HLLC: $t \approx 9.0$

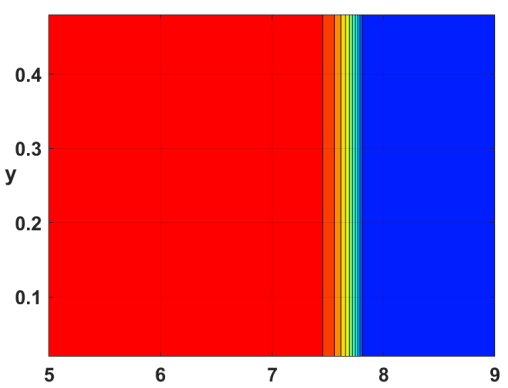

(g) Rotated-hybrid HLL/HLLC: $t \approx 9.0$

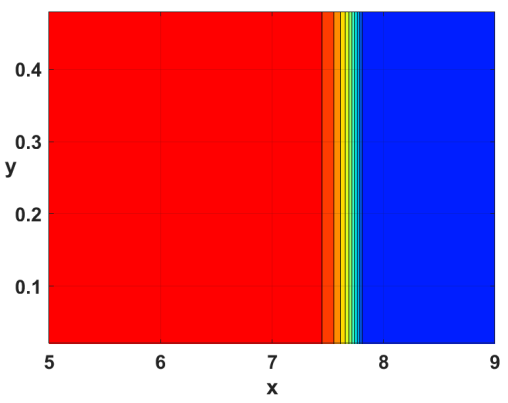

(j) Rotated-hybrid Rusanov/HLLC: $t \approx 9.0$

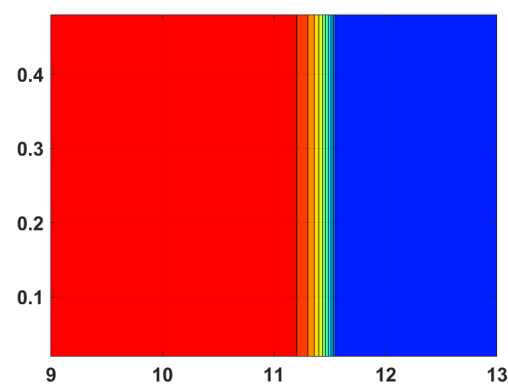

(b) Standard HLL: $t \approx 13.5$

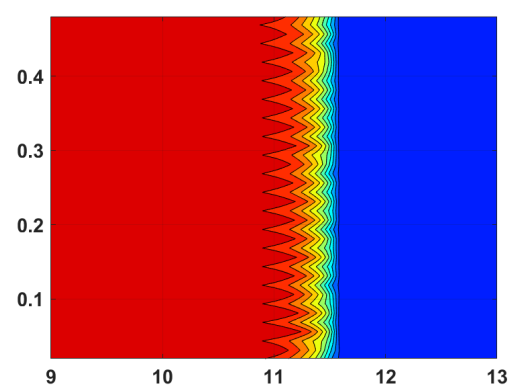

(e) Standard HLLC: $t \approx 13.5$

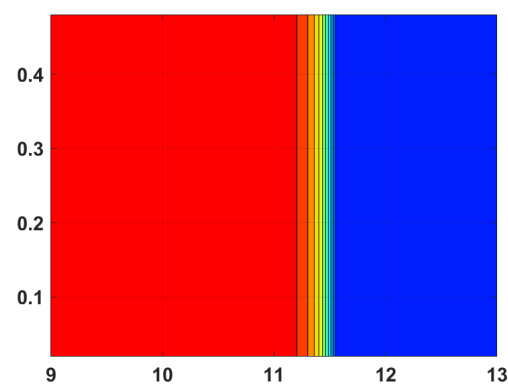

(h) Rotated-hybrid HLL/HLLC: $t \approx 13.5$

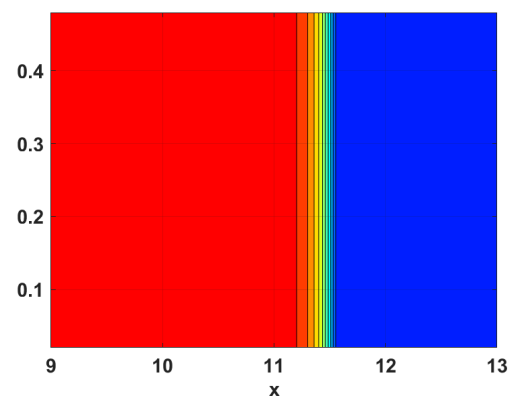

(k) Rotated-hybrid Rusanov/HLLC: $t \approx 13.5$

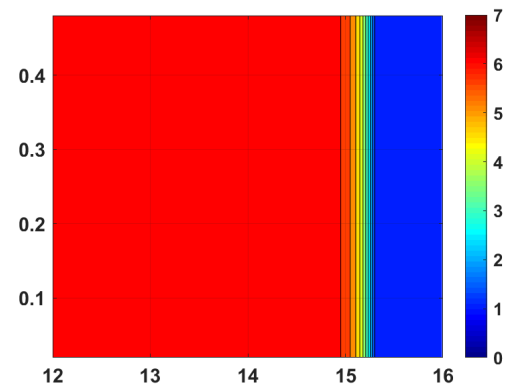

(c) Standard HLL: $t \approx 18.0$

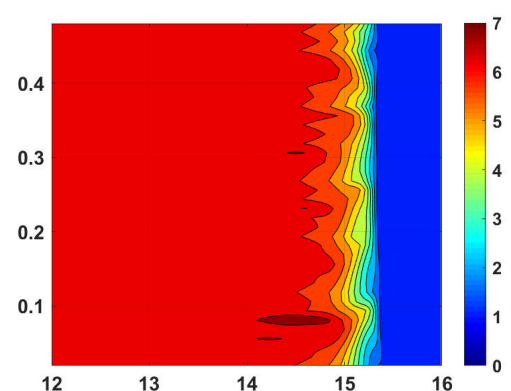

(f) Standard HLLC: $t \approx 18.0$

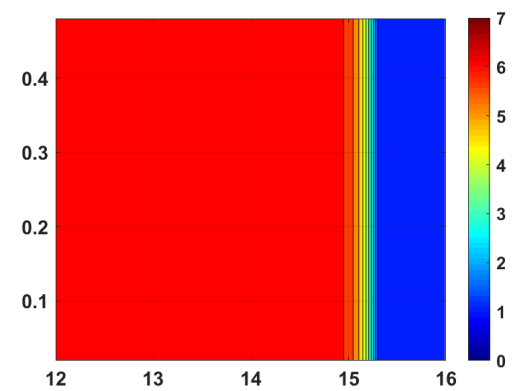

(i) Rotated-hybrid HLL/HLLC: $t \approx 18.0$

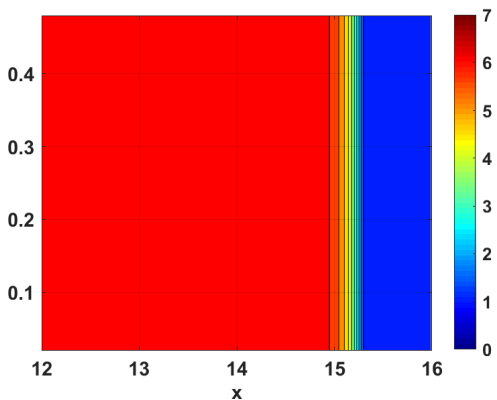

(1) Rotated-hybrid Rusanov/HLLC: $t \approx 18.0$

Figure 2. Rest-mass density contours of the odd-even decoupling using 10 contour levels a various simulation times using a first-order spatial reconstruction scheme. The discretisations consists of $800 \times 40$ cells. The Riemann solvers are: (2a)-(2c) standard HLL, (2d)-(2f) standard HLLC, (2g)-(2i) rotated-hybrid HLL/HLLC and (2j)-(2l) rotated-hybrid Rusanov/HLLC.

rotated-hybrid Rusanov/HLLC and HLL/HLLC Riemann solvers. The HLLC Riemann solver in figure 5c shows a clear protrusion jetting out of the growing RMI spike which is attributed to the Carbuncle phenomenon. This protrusion is absent in the HLL Riemann solver simulation seen in figure $5 \mathrm{~b}$ and for the rotated-hybrid HLL/HLLC simulation in figure 5e. This demonstrates the suppression of the Carbuncle problem when the more dissipative HLL
Riemann solver or the rotated-hybrid approach is used, similar conclusions can be seen from the Rusanov and rotated-hybrid $\mathrm{Ru}-$ sanov/HLLC Riemann solvers in figures $5 \mathrm{a}$ and $5 \mathrm{~d}$, respectively. It is possible that due to the protrusion emanating from the spike head an inaccurate growth-rate of the instability may be reported. The height of the instability, subsequently used to compute the growthrate, is defined as the extent of the mixing region with respect to 


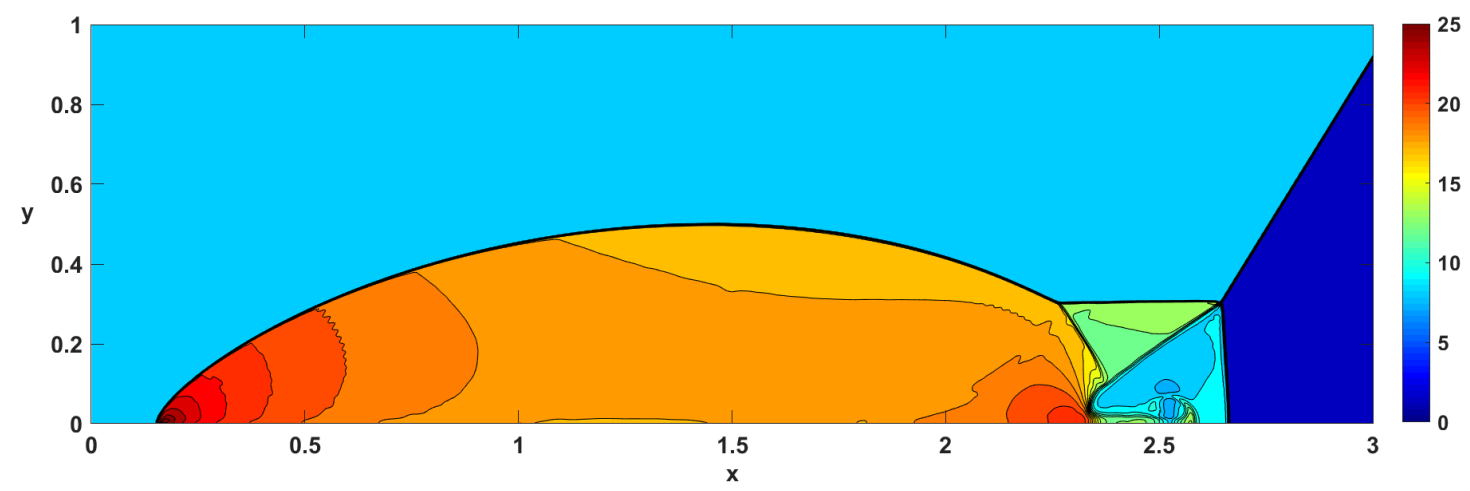

(a) Full solution using the standard HLL Riemann solver, the computational domain shown has size $[0,3] \times[0,1]$.

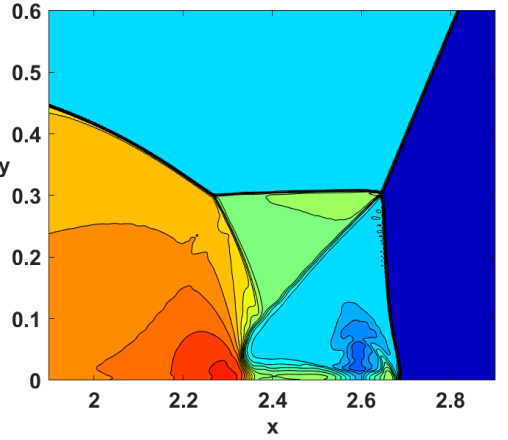

(b) Standard HLLC

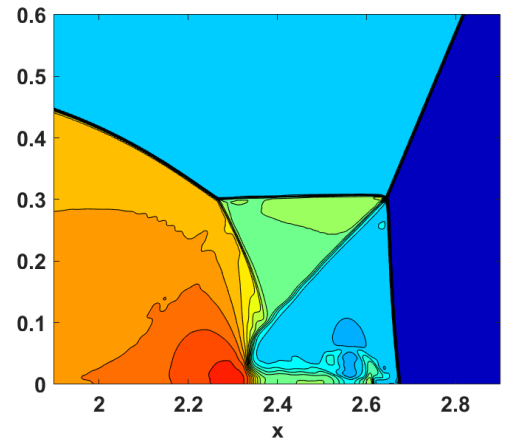

(c) Rotated-hybrid HLL/HLLC

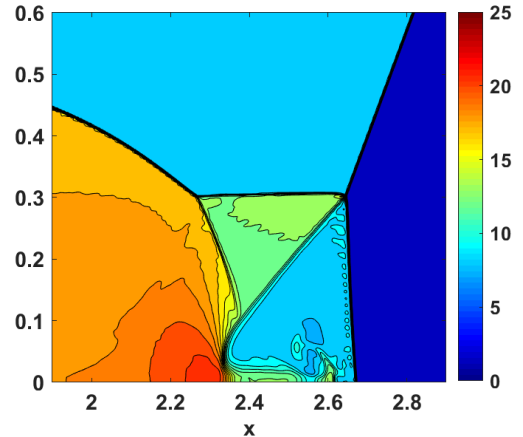

(d) Rotated-hybrid Rusanov/HLLC

Figure 3. Rest-mass density contours of the relativistic double Mach reflection using 25 contour levels at $t=4$. The WENO5 spatial reconstruction scheme is deployed and the discretisation consists of $1600 \times 400$ computational cells. The Riemann solvers shown are the: HLL (3a), HLLC (3b), rotated-hybrid HLL/HLLC (3c) and the rotated-hybrid Rusanov/HLLC (3d).

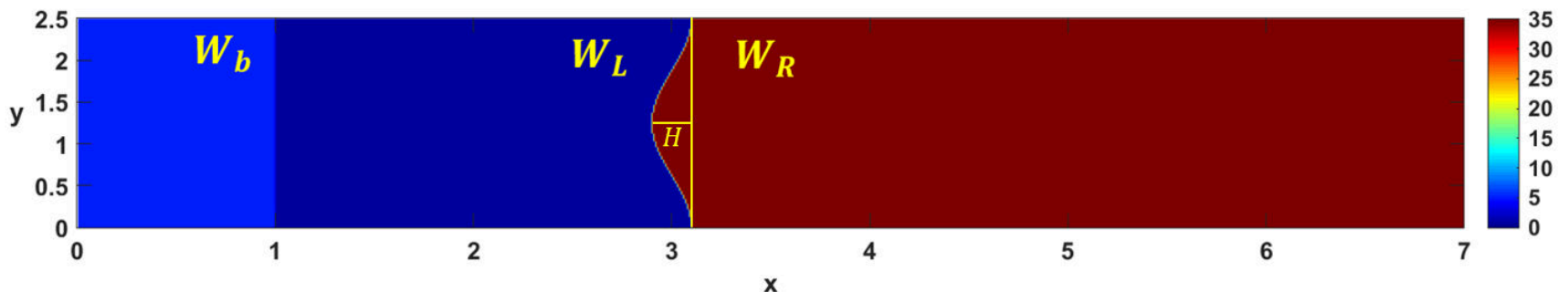

Figure 4. Schematic diagram for the relativistic Richtmyer-Meshkov instability initial conditions showing the rest-mass density. $\mathbf{W}_{b}$ denotes the initial primitive variables behind the rightward propagating shock, $\mathbf{W}_{L}$ and $\mathbf{W}_{R}$ are the initial conditions to the left and right of the initial perturbation, respectively. The profile of the perturbed interface is given by equation (44) and initial conditions for the three regions are specified in equation (45). The height $H$ is used to compute the perturbation amplitude evolution in figure 6

the moving interface, this is denoted as $H$ in figure 5, Zanotti \& Dumbser (2015). By this definition, the growth evolution over time for all cases is shown in figure 6. Note that for the HLLC Riemann solver a larger value of perturbation amplitude is found as the growth of the instability becomes non-linear, i.e. after $t \approx 15$. This is attributed to the spike protrusion as well as the oscillatory interface which are likely to corrupt the computation of the perturbation height. The inset of figure 6 shows a grid study in which the HLL Riemann was used at coarse, medium and fine resolutions, $750 \times 200,1500 \times 400$ and $3000 \times 800$, respectively. Note that for the coarser resolution the perturbation height falls marginally shorter when compared to the two finer resolutions which are in good agreement with one another. From this it is concluded that the resolution used to compare the HLL, HLLC and rotated-hybrid
Riemann solvers is sufficient and that the HLLC Riemann solver over-predicts the perturbation growth due to this numerical artefact. Furthermore, note that good agreement is found between the HLL and rotated-hybrid HLL/HLLC Riemann solvers, both of which are Carbuncle-free. To further support this statement, the evolution of the perturbation amplitude from the numerical simulations of Zanotti \& Dumbser (2015) is also displayed in figure 6. This data is in good agreement with the Carbuncle-free results in the linear growth stages and subsequent transition to the non-linear growth stage.

Another feature differing between each of the Riemann solver formulations apparent in figure 5 is the typical "mushroom" structure of the RMI. This structure appears more diffusive in the case of the HLL, whereas in the HLLC case the structure is somewhat unorganised as smaller-scale structures appear in the solution. A similar 
discussion may be found in Radice \& Rezzolla (2012) in the context of the relativistic Kelvin-Helmholtz instability where it has been shown that additional flow features may appear when schemes with less numerical dissipation are used. Similar conclusions have also been drawn by Mignone et al. (2009) and Beckwith \& Stone (2011) in the magnetised version. The rotated-hybrid HLL/HLLC formulation however presents a result giving greater detail in the RMI mushroom but without the disorganised smaller-scale structures providing a contribution to the rest-mass density field. To quantify the power residing at the smaller scales and the inherent numerical viscosity of the schemes, the rest-mass density power spectrum is reported for each case following Beckwith \& Stone (2011) and Radice \& Rezzolla (2012), to which the reader is referred to for the method of analysis used herein. Figure 7 displays the power spectra of the rest-mass density for the Rusanov, HLL, HLLC, rotated-hybrid Rusanov/HLLC and rotated-hybrid HLL/HLLC Riemann solver formulations by applying the Fourier transform in the $y$-direction, then computing the integrated power spectrum in the $x$-direction. The standalone Rusanov and HLL Riemann solver demonstrates the largest amount of numerical dissipation whereas the HLLC reports the least. The rotated-hybrid Rusanov/HLLC and HLL/HLLC then fall in-between the HLLC and Rusanov/HLL however they are less diffusive at higher wavenumbers than the Rusanov and HLL implementations. This result supports the idea that the rotated-hybrid approach is in general more dissipative but is still able to resolve small-scale structures where required. The alternative definition of the vector $\mathbf{n}_{1}$ using equation (29) is also shown in figure $5 \mathrm{f}$ along with its corresponding density power spectrum in figure 7 . While the interface separating the light and heavy fluid at the base of the instability is less sharp, the schemes perform in a very similar manner both in terms of its qualitative outcome and density power spectrum.

\subsection{Propagation of a Relativistic Jet}

As a final example, an application of the rotated-hybrid Riemann solver to simulate the propagation of relativistic jets is proposed. The initial conditions closely follow those described by Nunez-de la Rosa \& Munz (2016) and are summarised as follows. A computational domain of size $[0,15] \times[-15,15]$ is used where at $x=0$ the relativistic jet is injected into the domain through a nozzle of radius $r_{b}$ with a beam velocity $v_{b}$. The nozzle radius $r_{b}$ at the left boundary is centred around $y=0$ with the nozzle inlet described by $\left|y / r_{b}\right|<1$ where $r_{b}=0.2$. Outflow boundary conditions are applied everywhere in the domain except at the plane $x=0$ in which a reflecting boundary condition is used surrounding the injection zone. The initially homogeneous ambient medium is given conditions

$\mathbf{W}=(1.0,0.0,0.0,0.001)^{T}$

and the inflow boundary condition representing the jet nozzle is given conditions

$\mathbf{W}_{b}=(0.01,0.999,0.0,0.001)^{T}$

with a constant adiabatic index of $\Gamma=5 / 3$. Therefore, the ratio of rest-mass density of the beam fluid and ambient medium is $\eta=0.01$ and the ratio of pressure of the beam fluid and ambient medium is $\kappa=1$. The simulation is run for a time of $t \approx 20$ using a WENO3 spatial reconstruction scheme that reduces to a first-order spatial reconstruction in the vicinity of shocks, detected via equation (11). This choice was made to guarantee a stable solution in the case of
Table 1. Normalised CPU time for all presented simulations: A: ShockInstability, B: Double-Mach Reflection, C: Richtmyer-Meshkov, D: Relativistic Jet.

\begin{tabular}{ccccc}
\hline Case & No. of Cells & HLL & HLLC & HLL/HLLC \\
\hline A & $800 \times 40$ & 1 & 1.79 & 2.10 \\
B & $1600 \times 400$ & 1 & 1.24 & 1.36 \\
C & $1500 \times 400$ & 1 & 1.20 & 1.48 \\
D & $600 \times 600$ & 1 & 1.16 & 1.37 \\
\hline
\end{tabular}

the HLLC Riemann solver to provide a comparison with the other Riemann solver strategies.

Figure 9 contains the logarithm of the rest-mass density contours for all schemes at a resolution of $600 \times 600$ cells in figures $8 \mathrm{a}-8 \mathrm{c}$ for the HLL, HLLC and rotated-hybrid HLL/HLLC, respectively. A higher resolution of $1200 \times 1200$ for the rotated-hybrid HLL/HLLC is shown in figure 9a. The HLL Riemann solver in figure 8 a reproduces a diffused jet structure likely caused by the diffusive nature of the Riemann solver itself and the first-order scheme applied to shocked regions. The HLLC Riemann solver in figure $8 \mathrm{~b}$ on the other hand reproduces a much narrower jet that propagates further in the same time. A protrusion is also visible at the jet head as seen in the case of the Richtmyer-Meshkov instability case in section 6.3 which is again attributed to the Carbuncle phenomenon and deemed non-physical. When the rotated-hybrid HLL/HLLC approach is used, see figure $8 \mathrm{c}$, the protrusion is absent. Furthermore, a more detailed inner structure of the jet is visible when compared to the HLL Riemann solver in figure 8a, likely due to the reduction in numerical dissipation provided by the HLLC Riemann solver and witnessed in the rest-mass density power spectra analyses reported for the Richtmyer-Meshkov instability in figure 7. A test is also conducted to determine if such a protrusion may occur at larger resolutions. Figure 9a shows the rotated-hybrid HLL/HLLC simulation at twice the resolution of $1200 \times 1200$ cells in which the Carbuncle problem in the form of a protrusion is absent. The overall structure and morphology of the jet has changed as expected which is also observed by Nunez-de la Rosa \& Munz (2016) in their resolution study. Figures $9 \mathrm{a}$ and $9 \mathrm{~b}$ show the effect of changing the definition of the vector $\mathbf{n}_{1}$ using equation (27) and (29), respectively, for which negligible qualitative difference is found.

\subsection{Computational Timings}

Here the normalised computational efforts required for each case previously discussed and the chosen Riemann solver formulation is summarised in table 1 . The odd-even decoupling problem reports a much longer CPU time when the HLLC and rotated-hybrid HLL/HLLC Riemann solver are used, approximately twice as long for the latter. The remainder of the test cases report about a $25 \%$ increase in computational effort for the rotated-hybrid HLL/HLLC strategy when compared to the standalone HLLC Riemann solver.

\section{CONCLUSIONS}

The relativistic HLLC Riemann solver has been shown to exhibit numerical pathologies associated with the Carbuncle phenomenon through theoretical analysis and numerical test cases. To overcome this problem, a rotated-hybrid Riemann solver using the hybridisation of the HLL and HLLC approximate Riemann solvers (or the Rusanov and HLLC) has been proposed in the present work 


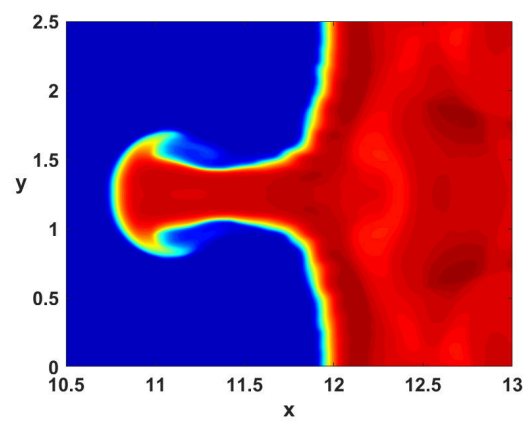

(a) Standard Rusanov

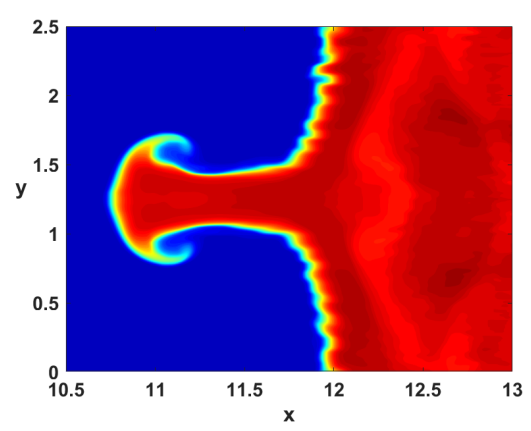

(d) Rotated-hybrid Rusanov/HLLC

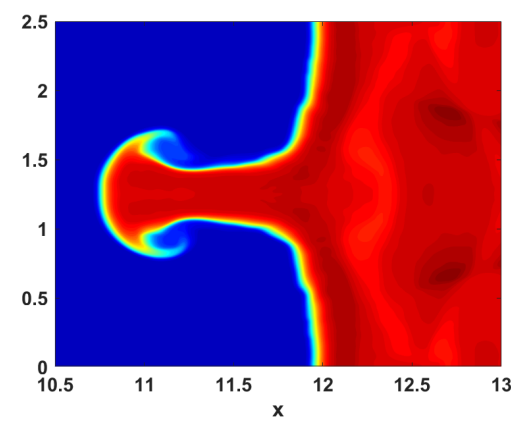

(b) Standard HLL

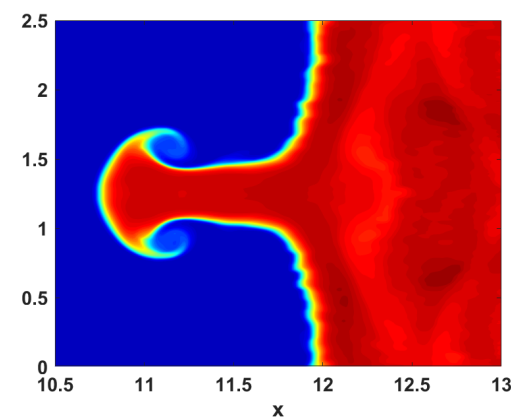

(e) Rotated-hybrid HLL/HLLC using the definition of $\mathbf{n}_{1}$ in equation (27).

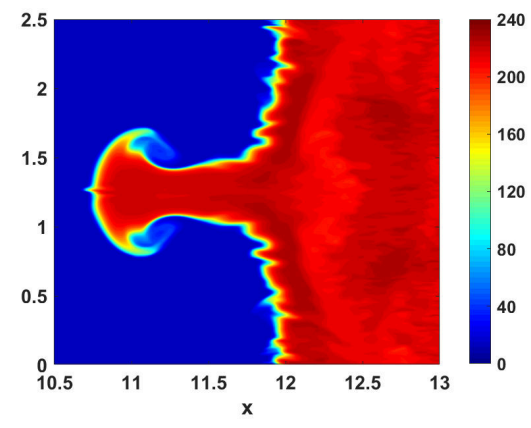

(c) Standard HLLC

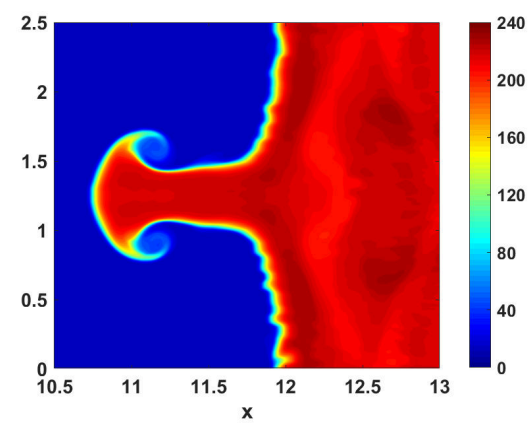

(f) Rotated-hybrid HLL/HLLC using the definition of $\mathbf{n}_{1}$ in equation (29).

Figure 5. Rest-mass density contours of the relativistic Richtmyer-Meshkov instability at $t \approx 30$ using the WENO5 spatial reconstruction scheme. The computational domain shown has size $[10.5,13] \times[0,2.5]$. The discretisations consists of $1500 \times 400$ cells. The Riemann solver used are: $(5 a)$ standard Rusanov, (5b) standard HLL, (5c) standard HLLC, (5d) rotated-hybrid Rusanov/HLLC and (5e-5f) rotated-hybrid HLL/HLLC using the definition of $\mathbf{n}_{1}$ in equation (27) and (29), respectively.

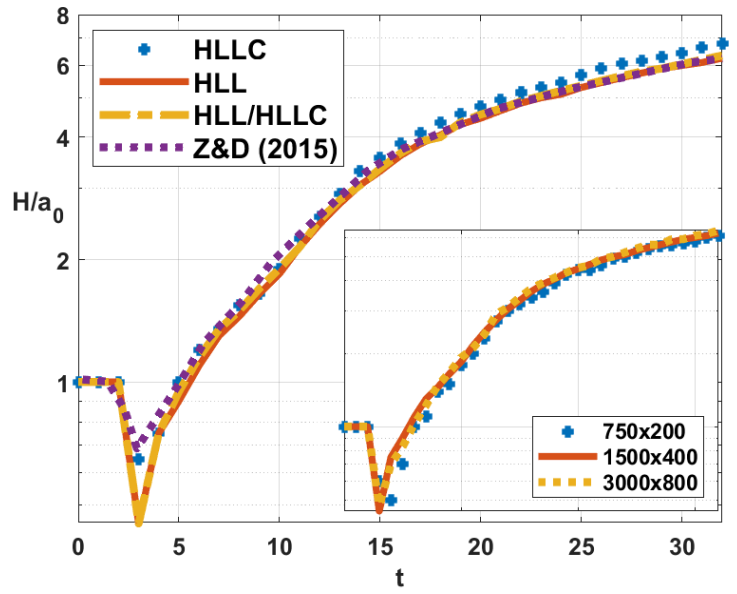

Figure 6. Time evolution for the amplitude in initial perturbation for the HLL, HLLC and rotated-hybrid HLL/HLLC Riemann solvers and comparison to numerical data achieved in Zanotti \& Dumbser (2015), [Z\&D (2015)]. Inset: time evolution for the amplitude in initial perturbation for the HLL Riemann solver at grid resolutions $750 \times 200,1500 \times 400$ and $3000 \times 800$.

for solving relativistic hydrodynamics problems. In the case of the numerical shock instability problem (the odd-even decoupling problem), due to the fact that the HLLC scheme has not been used in the direction of the shock, the rotated-hybrid scheme provides enough numerical dissipation such that perturbations in the pressure field do not grow over time to distort the rest-mass density field. There-

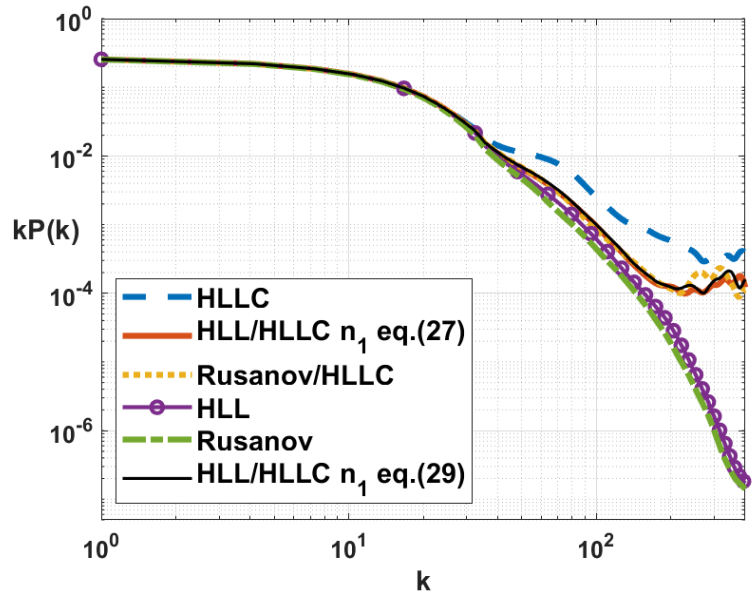

Figure 7. One-dimensional power spectrum of the rest-mass density for the HLL, HLLC and rotated-hybrid HLL/HLLC Riemann solvers at $t \approx 30$ using a grid resolution of $1500 \times 400$ cells.

fore, the proposed rotated-hybrid scheme does not produce spurious numerical artefacts. In the case of the double-Mach reflection, the kinked Mach stem phenomenon does not appear in the numerical solution when the rotated-hybrid Riemann solver is employed but it does exist when the standalone HLLC Riemann solver is used. The proposed rotated-hybrid Riemann solver provides a robust numerical framework to consistently predict the perturbation growth of the relativistic Richtmyer-Meshkov instability. This is 


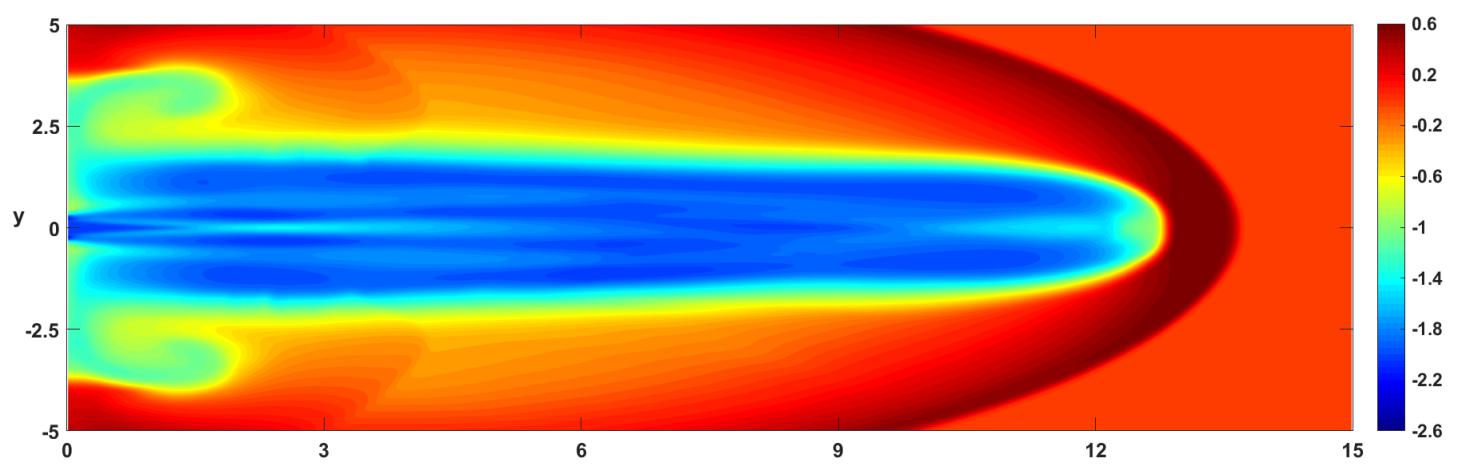

(a) Standard HLL Riemann solver using $600 \times 600$ cells.

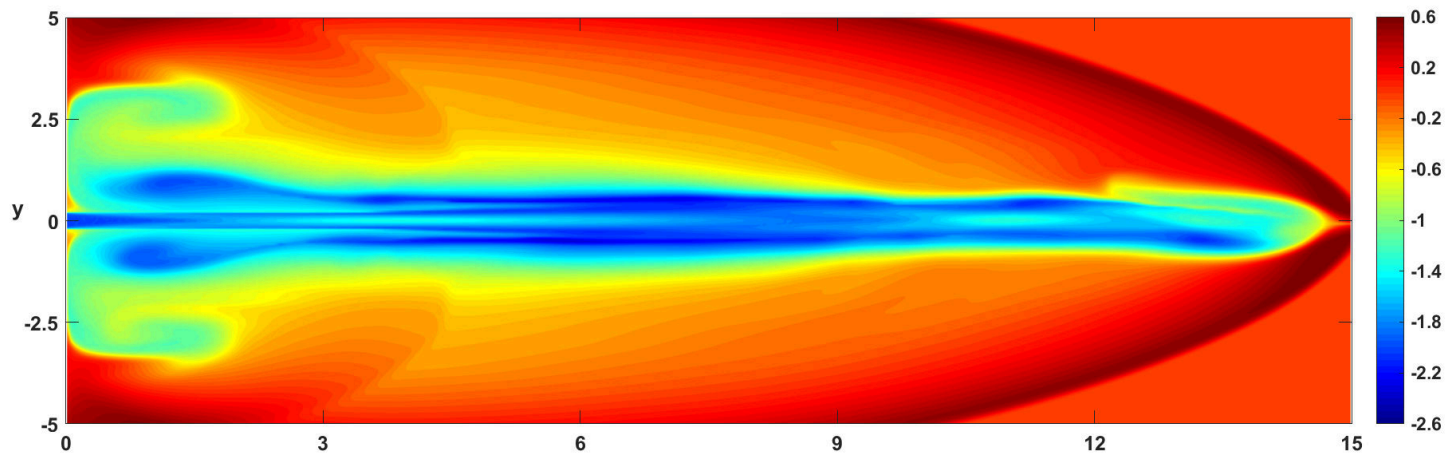

(b) Standard HLLC Riemann solver using $600 \times 600$ cells.

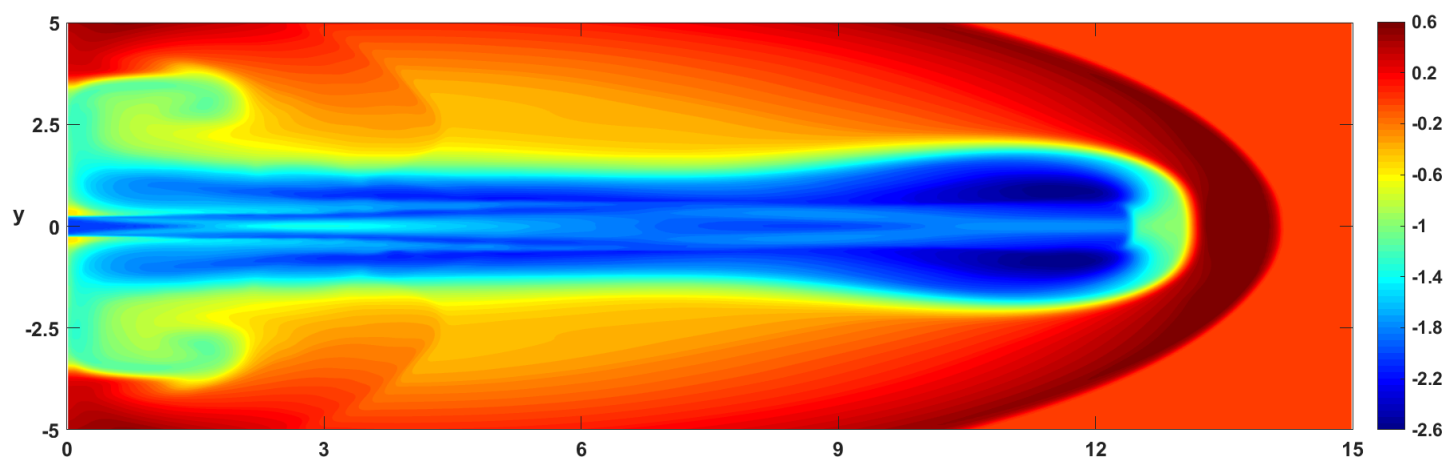

(c) Rotated-hybrid HLL/HLLC Riemann solver using $600 \times 600$ cells.

Figure 8. Logarithm of the rest-mass density contours for the relativistic jet at $t \approx 20$. The WENO3 spatial reconstruction scheme is used, reducing to a first-order reconstruction in the vicinity of shocks. The Riemann solvers used are: HLL (8a), HLLC (8b) and the rotated-hybrid HLL/HLLC (8c) at a grid resolution of $600 \times 600$ cells. Note that only a portion of domain is shown in the $y$-direction: $[0,5]$.

predominantly due to the elimination of the Carbuncle problem which causes a non-physical protrusion in the evolution of the spike tip. A resolution study using the stable HLL Riemann solver was conducted to determine a grid independent evaluation of the perturbation growth. It was then found that due to the Carbuncle problem an over-prediction in the growth was found when using the HLLC Riemann solver as the perturbation height is determined by taking the distance from the tip of the spike to the base of the bubble. Furthermore, the rotated-hybrid approach demonstrated the ability to better resolve smaller-scale structures than the standalone Rusanov or HLL Riemann solvers which was quantified via the use of the rest-mass density power spectrum. In the case of the relativistic jet propagation, it has been observed that the use of the rotated-hybrid
HLL/HLLC Riemann solver eliminates the non-physical Carbuncle protrusion at the head of the jet. In addition, a more detailed inner structure of the jet is visible when compared to the HLL Riemann solver, likely due to the reduction in numerical dissipation witnessed in the rest-mass density power spectrum analysis reported for the Richtmyer-Meshkov instability. Finally, it is shown that the choice of normal vector $\mathbf{n}_{1}$ used in defining the rotated-hybrid flux has negligible impact on the final solution when either choice is made. In addition to the oscillation-free behaviour of the proposed rotated-hybrid scheme, the computational time is increased compared to the standalone HLLC Riemann solver by roughly $25 \%$ for the astrophysical test cases when compared to the HLLC Riemann solver. Overall, this strategy is problem-independent, straightfor- 


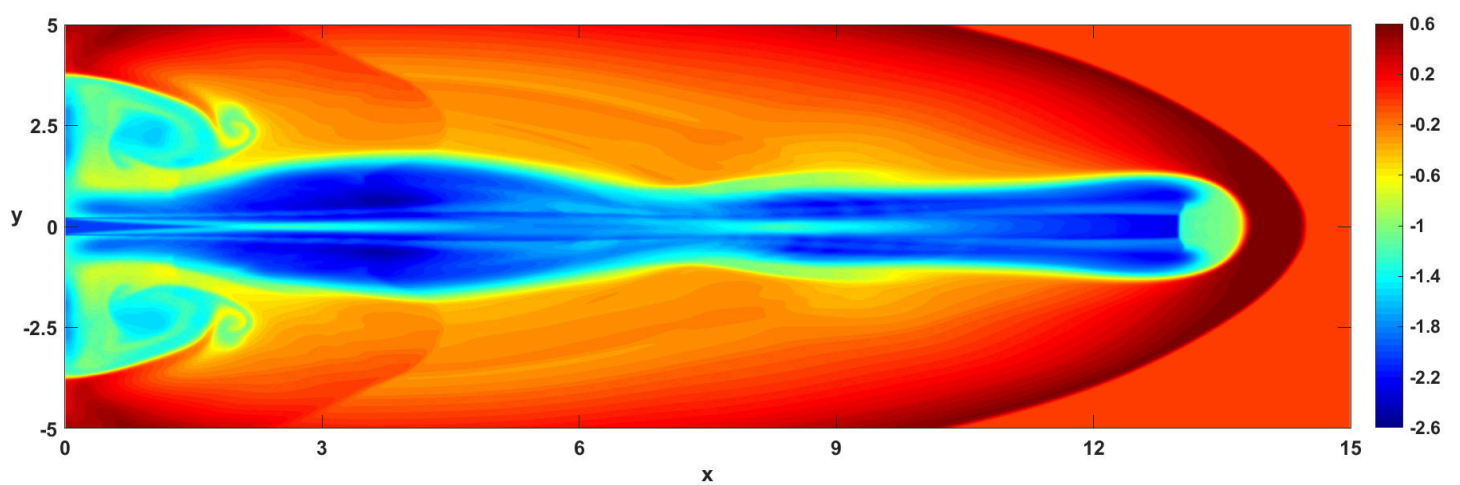

(a) Rotated-hybrid HLL/HLLC Riemann solver using $1200 \times 1200$ cells using the definition of $\mathbf{n}_{1}$ in equation (27).

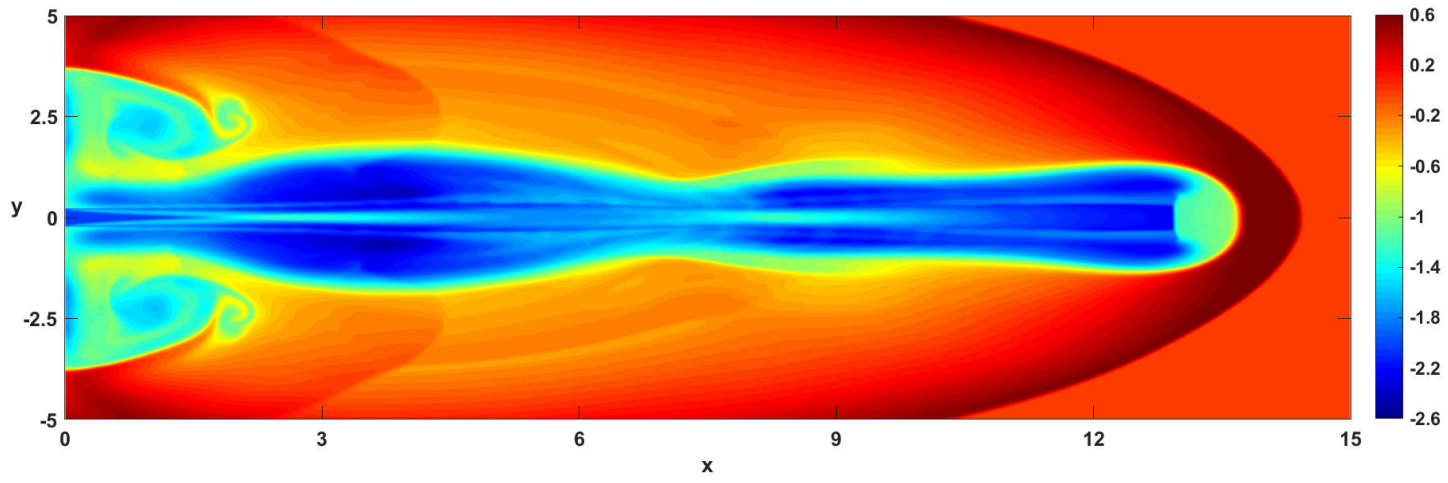

(b) Rotated-hybrid HLL/HLLC Riemann solver using $1200 \times 1200$ cells using the definition of $\mathbf{n}_{1}$ in equation (29).

Figure 9. Logarithm of the rest-mass density contours for the relativistic jet at $t \approx 20$. The WENO3 spatial reconstruction scheme is used, reducing to a first-order reconstruction in the vicinity of shocks. The Riemann solver used is the rotated-hybrid HLL/HLLC (9a-9b) using a finer resolution $1200 \times 1200$ cells and using the definitions for the vector $\mathbf{n}_{1}$ in equations (27) and (29), respectively. Note that only a portion of domain is shown in the $y$-direction: [0, 5].

ward to implement and provides a robust numerical solution when combined with Godunov-type high-order schemes for relativistic hydrodynamics. Finally, the authors note that this strategy could also be applied in the context of RMHD without any modification to the general rotated-hybrid methodology.

\section{ACKNOWLEDGEMENTS}

The present research work was financially supported by the Centre for Computational Engineering Sciences at Cranfield University under the project code EEB6001R. The authors would also like to thank Tom-Robin Teschner for his valuable comments during the preparation of the manuscript.

\section{DATA AVAILABILITY STATEMENT}

All data incorporated into the present article is available upon request. The data underlying this article will be shared on reasonable request to the first author of the present work (E-mail: j.townsend@cranfield.ac.uk).

\section{REFERENCES}

Balsara D. S., 2017, Living Reviews in Computational Astrophysics, 3, 2 Beckwith K., Stone J. M., 2011, The Astrophysical Journal Supplement Series, 193, 6
Davis S. F., 1984, Journal of Computational Physics, 56, 65

Einfeldt B., 1988, SIAM Journal on Numerical Analysis, 25, 294

Eulderink F., Mellema G., 1995, Astronomy \& Astrophysics Supplement Series, 110, 587

Gottlieb S., Shu C.-W., 1998, Mathematics of Computation of the American Mathematical Society, 67, 73

Harten A., Lax P. D., van Leer B., 1983, SIAM Review, 25, 35

Inoue T., 2012, Astrophysical Journal, 760, 0

Jameson A., Schmidt W., Turkel E., 1981, in 14th Fluid and Plasma Dynamics Conference, AIAA-81-1259.

Kane J., Drake R., Remington B., 1999, The Astrophysical Journal, 20, 335

Kim S. D., Lee B. J., Lee H. J., Jeung I. S., 2009, Journal of Computational Physics, 228, 7634

Kim S. D., Lee B. J., Lee H. J., Jeung I. S., Choi J.-Y., 2010, International Journal for Numerical Methods in Fluids, 62, 1107

Levy D. W., Powell K. G., van Leer B., 1993, Journal of Computational Physics, 106, 201

Liou M. S., 2000, Journal of Computational Physics, 160, 623

Maccormack R. W., 2011, 49th AIAA Aerospace Sciences Meeting including the New Horizons Forum and Aerospace Exposition, pp 1-13

Martí J., Müller E., 1994, Journal of Fluid Mechanics, 258, 317

Martí J. M., Müller E., 1996, Journal of Computational Physics, 123, 1

Martí J. M., Müller E., 2015, Living Reviews in Computational Astrophysics, 1,182

Matsumoto J., Masada Y., 2013, Astrophysical Journal Letters, 772

Meshkov E. E., 1972, Fluid Dynamics, 4, 101

Mignone A., Bodo G., 2005, Monthly Notices of the Royal Astronomical Society, 364, 126

Mignone A., Bodo G., 2006, Monthly Notices of the Royal Astronomical Society, 368, 1040 
Mignone A., Ugliano M., Bodo G., 2009, Monthly Notices of the Royal Astronomical Society, 393, 1141

Mohseni F., Mendoza M., Succi S., Herrmann H. J., 2014, Physical Review D - Particles, Fields, Gravitation and Cosmology, 90, 1

Nishikawa H., Kitamura K., 2008, Journal of Computational Physics, 227, 2560

Nunez-de la Rosa J., Munz C. D., 2016, Monthly Notices of the Royal Astronomical Society, 460, 535

Pons J. A., Martí J. M., Müller E., 2000, Journal of Fluid Mechanics, 422, 125

Quirk J. J., 1994, International Journal for Numerical Methods in Fluids, 18,555

Radice D., Rezzolla L., 2012, Astronomy \& Astrophysics, 547, A26

Ren Y. X., 2003, Computers and Fluids, 32, 1379

Rezzolla L., Zanotti O., 2001, Journal of Fluid Mechanics, 449, 395

Rezzolla L., Zanotti O., 2013, Relativistic Hydrodynamics, first edn. Oxford University Press

Richtmyer R. D., 1960, Communications on Pure and Applied Mathematics, 13, 297

Roe P. L., 1981, Journal of Computational Physics, 43, 357

Rusanov V. V., 1961, USSR Computational Mathematics and Mathematical Physics, 1, 267

Schneider V., Katscher U., Rischke D., Waldhauser B., Maruhn J., Munz C.-D., 1993, Journal of Computational Physics, 105, 92

Shu C.-W., 2009, SIAM Review, 51, 82

Simon S., Mandal J. C., 2019, Journal of Computational Physics, 378, 477

Titarev V. A., Toro E. F., 2004, Journal of Computational Physics, 201, 238

Toro E. F., Spruce M., Speares W., 1994, Shock Waves, 4, 25

Tsoutsanis P., 2019, Journal of Computational Physics: X, 4, 100037

Wang P., Abel T., Zhang W., 2008, The Astrophysical Journal Supplement Series, 176, 467

Woodward P. R., Colella P., 1984, Journal of Computational Physics, 54, 115

Zanotti O., Dumbser M., 2015, Physics of Fluids, 27

Zhang W., MacFadyen A. I., 2006, The Astrophysical Journal Supplement Series, 164, 255 


\section{On the development of a rotated-hybrid HLL/HLLC approximate Riemann solver for relativistic hydrodynamics}

Townsend, Jamie F.

Oxford University Press

Townsend JF, Könözsy L, Jenkins KW. (2020) On the development of a rotated-hybrid HLL/HLLC approximate Riemann solver for relativistic hydrodynamics. Monthly Notices of the Royal Astronomical Society, Volume 496, Issue 2, August 2020, pp. 2493-2505 https://doi.org/10.1093/mnras/staa1648

Downloaded from Cranfield Library Services E-Repository 